\title{
MILLI-ARCSECOND PROPER MOTIONS IN THE PLEIADES
}

\author{
E. SCHILBACH ${ }^{1}$, J. GUIBERT ${ }^{2}$ and J. SOUCHAY ${ }^{2}$ \\ ${ }^{1}$ Universität Potsdam/Sternwarte Babelsberg, An der Sternwarte 16, D-14482 \\ Potsdam, Germany \\ ${ }^{2}$ Centre d'Analyse des Images, Observatoire de Paris, 61 av. de l'Observatoire, \\ F-75014 Paris, France
}

\begin{abstract}
As a first step in our open cluster programme, a preliminary catalogue of proper motions and photographic B, V magnitudes for stars up to 18 mag within a region centred near Alcyone is presented. The catalogue is based on MAMA measurements of plates taken with the Tautenburg Schmidt telescope. A preliminary survey includes approx. 20,000 stars and covers a total field of about 9 square degrees. For stars brighter than $B=18 \mathrm{mag}$ an accuracy of $0.05 \mathrm{mag}$ and 2.5 mas/year has been estimated for photometric data and proper motions, respectively. The results of the determination of the Pleiades membership up to magnitude 18 are presented.
\end{abstract}

In the framework of our open cluster programme, a proper motion survey and a photographic BVR photometry up to the 18th magnitude covering a 16 square degree region located in the Pleiades is performed. For this purpose we use partly overlapping plates taken with the Tautenburg and CERGA Schmidt telescopes as well as plates of the Bonn double refractor and Carte du Ciel plates. A detailed description of the programme is given in Schilbach et al. (1992). In this paper we present proper motions and photographic $B, V$ photometry in the field of Pleiades derived from MAMA measurements of 8 from 24 Tautenburg plates provided for these investigations.

Each plate was transformed to the master plate using the method of stepwise regression with a complete third-order polynomial in powers of coordinates. As reference, faint anonymous stars with small proper motions and within a narrow range of magnitudes were selected. The rms of the mean proper motions of stars measured on at least three old and three new plates is 2.9 mas/year $(B<12), 1.9$ mas/year $(12<B<17)$ and 4.7 mas/year $(B>17)$.

The calibration of $B$ and $V$ plates was based on approx. 270 standards up to 19 mag taken from Johnson et al. (1958), Landolt (1979), Stauffer $(1980,1984)$. Magnitudes of measured stars were obtained by fitting the magnitudes and MAMA density fluxes of the standards. The internal accuracy of photographic magnitudes obtained from MAMA measurements of at least three plates of the same colour is $0.05 \mathrm{mag}(V<18), 0.08 \mathrm{mag}(V>17)$ and $0.04 \mathrm{mag}(B<18), 0.06 \mathrm{mag}$ $(B>18)$.

The astrometric and photometric data were used for the determination of the Pleiades membership. According to the proper motion vector point diagram, cluster members are clearly separated from the general field and show strong concentration to point +18.3 mas/year (RA), -41.9 mas/year (DEC). 520 cluster candidates were selected inside a circle with a radius of 12.5 
mas/year. Cluster membership probabilities were estimated with a criterion given in Ebbinghausen (1942). For each magnitude interval, the criterion based on a simple relation between coordinates of the cluster centroid $\left(\mu_{x 0}, \mu_{50}\right)$ proper motions $\left(\mu_{x}, \mu_{y}\right)$ and their mean errors $(\sigma)$ is given by:

1) for 'high probable' members: $\left(\mu_{x}-\mu_{x 0}\right)^{2}+\left(\mu_{y}-\mu_{50}\right)^{2} \leq 2 \sigma^{2}$;

2) for 'probable' members (2): $2 \sigma^{2} \leq\left(\mu_{x}-\mu_{x_{0}}\right)^{2}+\left(\mu_{y}-\mu_{50}\right)^{2} \leq 4 \sigma^{2}$;

3) for 'low probable' members : $4 \sigma^{2} \leq\left(\mu_{x}-\mu_{x 0}\right)^{2}+\left(\mu_{y}-\mu_{30}\right)^{2} \leq 8 \sigma^{2}$.

The probability to find cluster members outside the biggest circle is less than 2 percent.

Among all 520 candidates, 274, 150 and 60 stars were found to be 'high probable', 'probable' and 'low probable' members respectively. The colour magnitude diagram for all 520 candidates is plotted in Fig. 1. The same sample is shown in Fig. 2 as a function of absolute magnitudes $M_{V}$. The distance modulus of the Pleiades was assumed as $(m-M)_{0}=5.54 \mathrm{mag}$ with $A_{V}=0.12$ mag.

\section{Conclusion}

The achieved accuracy of proper motions and photographic photometry provides a reliable determination of the Pleiades membership up to $B=18$ mag.

The shift of faint Pleiades members $(V \geq 17.5 \mathrm{mag})$ to the left on the colour magnitude diagram may on the one hand indicate a white dwarf subsystem not found in the Pleiades until now. On the other hand, this shift may be caused by systematic errors of magnitude determination. There are only a few accurate standards fainter than $B=18 \mathrm{mag}$. The high dispersion of faint Pleiades members may be explained by a low accuracy of measurements as

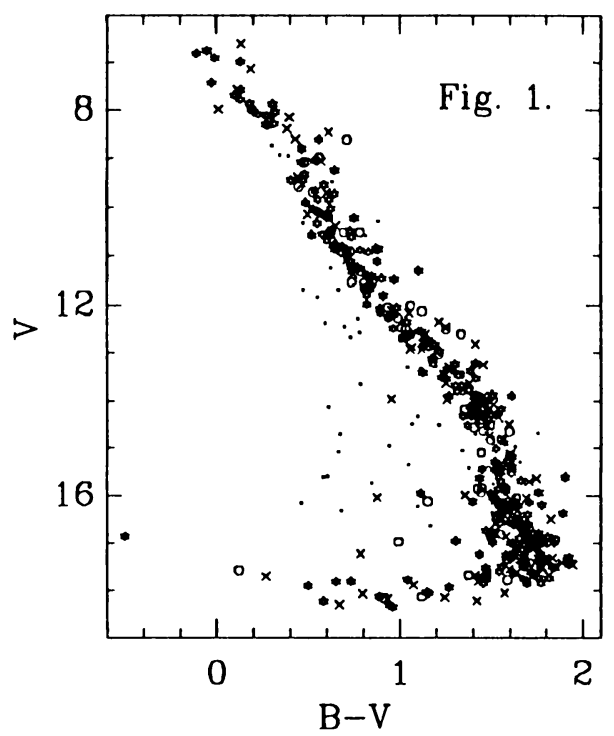

Figure 1. The colour-magnitude diagram for 520 cluster candidates. The 'high probable', 'probable' and 'low probable' members are indicated by stars, crosses and open clusters, respectively. 


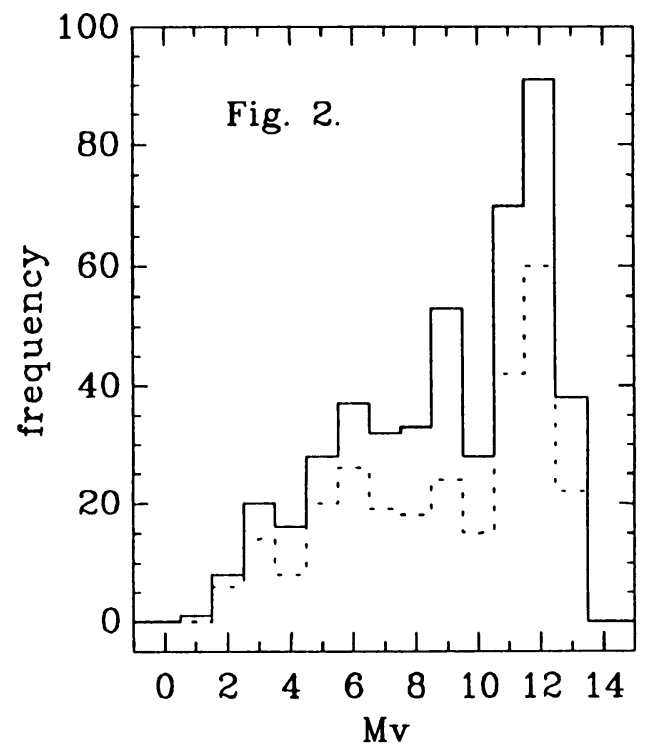

Figure 2. Distribution of cluster members [groups (1), (2) and (3)] versus their absolute magnitudes $M_{V}$ (solid line). The dotted line indicates the distribution for the 'high probable' members only.

well as by a probable false identification. The luminosity function derived from the data grows towards the peak at $M_{V}=12$. A decrease for fainter stars seems to be caused only by the limiting magnitude of the survey.

Whereas a small concentration of cluster members can be seen around the cluster centre, there are many remote candidates in outer parts of the field examined.

The preliminary survey is based on measurements of about 20 per cent of observational data we propose to use for the final catalogue. With all data, we expect to improve the accuracy of the survey as well as to extend the investigation to the outer parts of the Pleiades.

\section{References}

Ebbinghausen, E.G., 1942. Astron. J., 50, 1.

Johnson, H.L. and Mitchell, R.I., 1958. Astrophys. J., 128, 31.

Landolt, A.U., 1979. Astrophys. J., 231, 468.

Schilbach, E., Guibert, J., Geffert, M. and Hirte, S., 1993. In 'Developments in Astrometry and their Impacts on Astrophysics and Geodynamics', eds. Mueller et al., Reidel Publ. Comp., Dordrecht, p. 255.

Stauffer, J., 1982. Astron. J., 87, 1507.

Stauffer, J., 1984. Astrophys. J., 280, 189. 